\title{
A Rapid Radiochemical Purity Testing Method for ${ }^{99 \mathrm{~m} T c-T e t r o f o s m i n}$
}

\author{
Laura A. Eggert ${ }^{1}$, Michael D. Dick ${ }^{1}$, Douglas W. Mahoney ${ }^{2}$, Joseph P. Olson ${ }^{1}$, Gregory L. Werner ${ }^{1}$, and Joseph C. Hung ${ }^{1}$ \\ ${ }^{1}$ Division of Nuclear Medicine, Department of Radiology, Mayo Clinic, Rochester, Minnesota; and ${ }^{2}$ Division of Biomedical Statistics and \\ Informatics, Mayo Clinic, Rochester, Minnesota
}

\begin{abstract}
The standard radiochemical purity $(\mathrm{RCP})$ testing method for 99mTc-tetrofosmin as described in the package insert requires extensive time (20-30 min) and considerable skill to achieve accurate results. Additionally, the instant thin-layer chromatography strip impregnated with silica gel $(2 \times 20 \mathrm{~cm})$ used in the standard method will not be commercially available in the future. The purpose of this study was to evaluate whether a method developed by our laboratory for RCP testing of $99 \mathrm{mT}$-sestamibi could also be used as an alternative method for the RCP assay of ${ }^{99 m}$ Tc-tetrofosmin. Methods: The alternative RCP testing system consisted of a precut paper strip $(1 \times 8.5 \mathrm{~cm})$ from solvent saturation pads (Pall Corp.) as the stationary phase, with 1:1 chloroform:tetrahydrofuran used as the mobile phase. To validate the reliability of the alternative method, RCP values from 17 kit preparations were compared with the 2 methods. Kits were reconstituted according to the package insert instructions, and 4 additions of ${ }^{99 m} \mathrm{Tc}$-sodium pertechnetate were purposely added to create trials with RCP values below the accepted limit of $90 \%$ purity. Results: Two hundred four trials (100 of which were replicated) were run from the 17 kit preparations. Sixtyfour $(31 \%)$ of the 204 trials were below $90 \%$ purity based on the standard method. The overall agreement between the standard and alternative methods was $94 \%$ (192/204). The sensitivity of the alternative method for unacceptable RCP limits was $86 \%$ (55/64), and the specificity for acceptable RCP values was $98 \%$ (137/140). The agreement between the replicated trials of the alternative method was $99 \%(99 / 100)$, and for the standard method it was $92 \%(92 / 100)$. Conclusion: The standard method proved to be a much slower method and requires much more precision and attention. The alternative method is much faster, is easier, requires less attention to the solvent-development process, and can be used for RCP testing of both ${ }^{99 m}$ Tc-tetrofosmin and ${ }^{99 m}$ Tc-sestamibi. Furthermore, the stationary phase is much more readily available, is not moisture-sensitive, and is less susceptible to operator technique. Our method is accurate in deter-

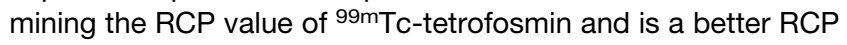
testing method for $99 \mathrm{mTc}$-tetrofosmin.
\end{abstract}

Key Words: 99mTc-tetrofosmin; radiochemical purity; quality control; nuclear pharmacy; radiopharmaceutical

J Nucl Med Technol 2010; 38:81-84

DOI: $10.2967 /$ jnmt.109.070573

Received Oct. 22, 2009; revision accepted Feb. 4, 2010.

For correspondence or reprints contact: Joseph C. Hung, Department of Radiology, Mayo Clinic, 200 First St. SW, Rochester, MN 55905-0001.

E-mail: jhung@mayo.edu

COPYRIGHT @ 2010 by the Society of Nuclear Medicine, Inc.
$\mathbf{T}$ he method suggested in the package insert for ${ }^{99 \mathrm{~m}} \mathrm{Tc}-$ tetrofosmin (Myoview; GE Healthcare) to test the radiochemical purity (RCP) of the radiopharmaceutical uses instant thin-layer chromatography (ITLC) impregnated with silica gel paper (ITLC-SG) (Pall Corp.) as the stationary phase and a 35:65 acetone:dichloromethane mixture as the mobile phase (Fig. 1) (1,2). Because the ITLC-SG paper will no longer be commercially available, along with several other disadvantages of this method (Table 1), an alternative method is necessary. An alternative method for the RCP testing of ${ }^{99 \mathrm{~m}}$ Tc-tetrofosmin using Whatman 1 paper (3) currently exists, but we found that it took considerably longer than the $3 \mathrm{~min}$ indicated in the article (3). Additionally, after we evaluated the method, we found that it had a high specificity of $95 \%$ but a sensitivity of only $70 \%$ relative to the standard method (Eggert et al., unpublished data, 2009).

An alternative RCP testing method for ${ }^{99 \mathrm{~m}} \mathrm{Tc}$-sestamibi, proposed by Hung et al. (4) in 1991, was evaluated for

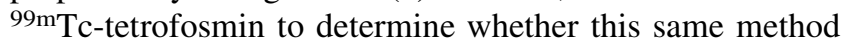
could be used for both reconstituted products. The proposed alternative method uses solvent saturation pads (Krackeler Scientific, Inc.) as the stationary phase and a 1:1 chloroform:tetrahydrofuran mixture as the mobile phase (Fig. 1). The purpose of this research was to determine whether the alternative method was a reliable and valid method for testing the RCP of ${ }^{99 \mathrm{~m}} \mathrm{Tc}$-tetrofosmin.

\section{MATERIALS AND METHODS}

\section{Standard RCP Testing Method for ${ }^{99 m}$ Tc-Tetrofosmin}

The manufacturer's suggestions call for ITLC-SG chromatography paper, which needs to be stored in a desiccator (1). The ITLCSG paper must be cut into strips measuring $2 \times 20 \mathrm{~cm}$ that are used as the stationary phase (1). Additionally, a 35:65 acetone:dichloromethane mixture is used as the mobile phase (Fig.1). The mixture should be poured into an ascending chromatography cylinder, which should be covered and allowed to equilibrate with the solvent vapor (1). The ITLC-SG strip must be marked accordingly to ensure accurate migration levels are achieved (Fig. 1).

A 10- to $20-\mu \mathrm{L}$ sample should be applied at the origin line using a $1-\mathrm{mL}$ syringe with a 28.5 -gauge needle. The strip should then be placed in the chromatography cylinder and covered 


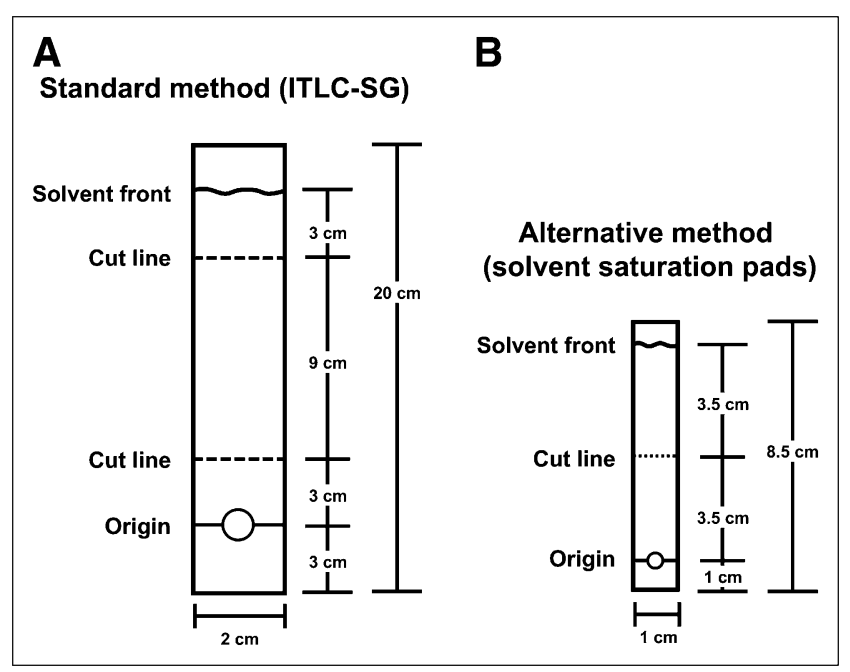

FIGURE 1. Chromatography diagrams of standard (A) and alternative (B) methods for RCP testing of $99 \mathrm{mTc}$-tetrofosmin.

immediately. To avoid any inaccuracies in the results, the strip should not be allowed to adhere to the walls of the cylinder. Once the solvent reaches the appropriate migration level, the strip should be removed from the tank and allowed to dry. The strip needs to then be cut into 3 pieces at the marked cut points. Each section should be measured separately using a dose calibrator to measure the activity present.

In this system, free ${ }^{99 \mathrm{~m}} \mathrm{Tc}$-sodium pertechnetate runs to the top portion of the strip, ${ }^{99 \mathrm{~m}} \mathrm{Tc}$-tetrofosmin migrates to the center portion of the strip, and hydrolyzed-reduced ${ }^{99 \mathrm{~m}} \mathrm{Tc}$, along with any hydrophilic complex impurities, remains at the origin in the bottom portion of the strip.

\section{Alternative RCP Testing Method for ${ }^{99 m}$ Tc-Tetrofosmin}

The alternative method calls for solvent saturation pads, measuring $1 \times 8.5 \mathrm{~cm}$, to be used as the stationary phase (Fig.1). The strip should be marked accordingly to ensure proper migration levels are achieved (Fig. 1). A $10-$ to $20-\mu \mathrm{L}$ sample must be placed at the origin using a 1-mL syringe with a 28.5-gauge needle. The sample strip should be placed into a blood collection tube (Kendall Monoject; Tyco Healthcare) with a 1:1 chloroform:tetrahydrofuran mixture acting as the mobile phase. The tube should be covered immediately after the introduction of the strip, and the strip should not be allowed to adhere to the walls of the tube. Once the solvent reaches the appropriate line, it should be removed from the chromatography tank and allowed to dry. On completion, the strip must be cut at the designated cut line, and the activity on each half of the strip should be measured using a dose calibrator.

With this system, free ${ }^{99 \mathrm{~m}} \mathrm{Tc}$-sodium pertechnetate, hydrolyzed-reduced ${ }^{99 \mathrm{~m}} \mathrm{Tc}$, and any hydrophilic complex impurities remain at the origin in the bottom portion of the strip, and ${ }^{99 \mathrm{~m}} \mathrm{Tc}-$ tetrofosmin runs to the top portion of the strip.

\section{Fabrication of Various RCP Levels of ${ }^{99 m}$ Tc-Tetrofosmin}

In determining whether the proposed alternative method was an accurate procedure, it was necessary to verify and compare the results of both the current standard ITLC-SG method and the proposed alternative method. Initially, each kit was prepared according to the package insert instructions (1). Using a shielded, sterile syringe, we added $8,880 \mathrm{MBq}(240 \mathrm{mCi})$ of ${ }^{99 \mathrm{~m}} \mathrm{Tc}$ generator eluate diluted to $8 \mathrm{~mL}$ into a shielded kit vial containing a venting needle. Before the syringe was removed from the vial, $2 \mathrm{~mL}$ of gas was withdrawn. The venting needle and syringe were then removed, and the vial was mixed gently for $10 \mathrm{~s}$ before being allowed to incubate at room temperature for $30 \mathrm{~min}$, rather than $15 \mathrm{~min}$ as recommended in the package insert (1). The room temperature in our laboratory is set at $20^{\circ} \mathrm{C}$ rather than $25^{\circ} \mathrm{C}$, because of the requirements of the United States Pharmacopeia (USP), General Chapter $<797>$ (5). The colder temperature may slow down the transchelation process (6) during the reconstitution of ${ }^{99 \mathrm{~m}} \mathrm{Tc}$-tetrofosmin, which was the reason that the $30-\mathrm{min}$ incubation period was used.

After the initial RCP testing after $30 \mathrm{~min}$ of incubation, ${ }^{99 \mathrm{~m}} \mathrm{Tc}-$ sodium pertechnetate was added to create preparations for trials with RCP values below $90 \%$ purity. A range of 296-444 MBq (8-12 $\mathrm{mCi})$ of ${ }^{99 \mathrm{~m}} \mathrm{Tc}$-sodium pertechnetate was added to the reconstituted kit for each trial, and 4 additions-each within this specified range of radioactivity-were completed for each kit. After each addition, the kit was mixed gently and allowed to incubate at room temperature for $10 \mathrm{~min}$ before RCP testing was completed (Fig. 2).

Seventeen kits were prepared, and 204 trials were run. Of those trials, 100 were replicates. For each trial, 2 strips for each RCP testing method were completed. The second strip for each method was considered the replicate of that trial. The data were summarized, and the sensitivity and specificity of each method were determined.

TABLE 1

Comparison Between Standard and Alternative RCP Testing Systems

\begin{tabular}{lc}
\multicolumn{1}{c}{ Factor } & Standard method \\
\hline Food and Drug Administration- and USP (2)-approved & Alternative method \\
Single-strip method & $\times$ \\
Paper commercially available & $\times$ \\
Short procedure time & $\times$ \\
Little experience required to master method & $\times$ \\
So influence of solvent migration on results & $\times$ \\
\hline$=$ pro $; \times=$ con. & $\times$ \\
\hline
\end{tabular}




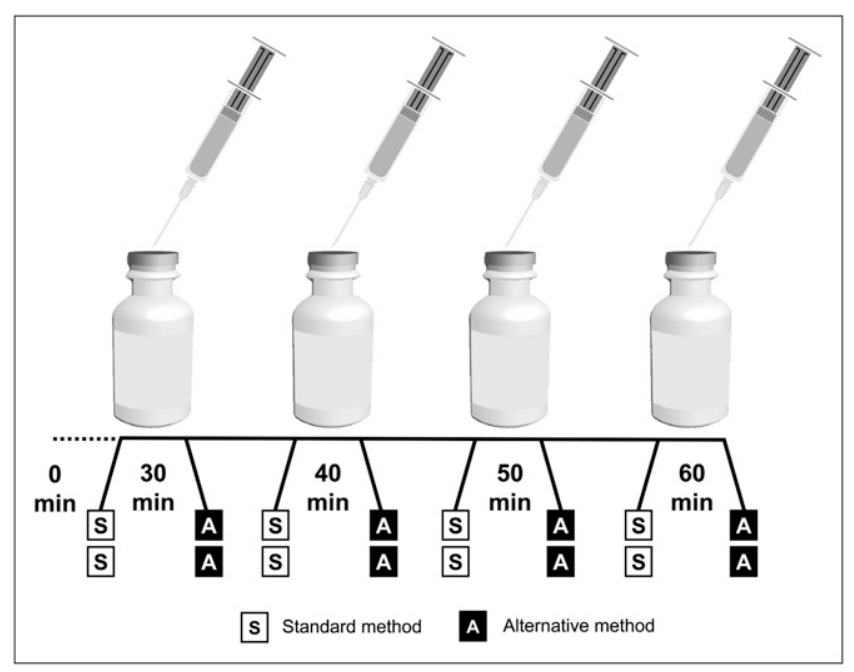

FIGURE 2. Two strips (initial strip and replicate) were run for both standard and alternative RCP testing methods at $30 \mathrm{~min}$ after initial reconstitution of kit at $0 \mathrm{~min}$. Additions of $99 \mathrm{mTC}$ sodium pertechnetate were made at $30,40,50$, and 60 min. After each addition, there was 10 min of incubation, followed by RCP testing using both methods. RCP evaluation was completed at $40,50,60$, and $70 \mathrm{~min}$, each being a time point for separate ${ }^{99 \mathrm{~m} T c}$-sodium pertechnetate addition.

\section{Comparison Between Standard and Alternative Testing Methods}

The RCP for each method was determined both before and after the fabrication of the kits. In doing this, 2 strips for each method were used to measure the RCP value of the prepared kit.

\section{RESULTS}

Fabrication of Various RCP Levels of ${ }^{99 m} \mathrm{Tc}-$ Tetrofosmin

The RCP of each reconstituted ${ }^{99 \mathrm{~m}}$ Tc-tetrofosmin vial ( $n=17$ ) was evaluated before and after the additions of ${ }^{99 \mathrm{~m}} \mathrm{Tc}$-sodium pertechnetate. ${ }^{99 \mathrm{~m}} \mathrm{Tc}$-sodium pertechnetate was added to generate a range of RCP values above and below the acceptable limits-values that were grouped as 60-89\% $(n=64), 90-94 \%(n=61)$, and 95-100\% $(n=$ 79). For evaluation purposes, a passing value for the radiochemical purity was greater than $90 \%$ and a failing value less than $90 \%(1)$.

\section{Comparison Between Standard and Alternative Testing Methods}

From the 17 kit preparations, 204 trials were conducted. Of those, 100 trials were replicates of the same kit preparation at each fabrication level. Of the total 204 trials, 64 (31\%) were below the $90 \%$ purity based on the standard method.

For the alternative method, the sensitivity for unacceptable RCP values (below $90 \%$ purity) was $86 \%$ (55/64). Additionally, the specificity for acceptable RCP values (greater than $90 \%$ purity) was $98 \%$ (137/140). The overall agreement between the standard and the alternative methods was $94 \%$ (192/204). The agreement between the replicated trials of the alternative method was $99 \%$ (99/100), and for the standard method it was $92 \%(92 / 100)$.

\section{DISCUSSION}

${ }^{99 m}$ Tc-tetrofosmin is an important radiopharmaceutical in the assessment of regions of reversible myocardial ischemia and the evaluation of ventricular function $(7,8)$. Because of the incubation period during the preparation of the ${ }^{99 \mathrm{~m}}$ Tc-tetrofosmin, a rapid RCP method is necessary to ensure the most prompt availability possible. The lengthy RCP method currently used, taking 20-30 min, is unacceptable in the fast-paced environment of nuclear pharmacy and nuclear medicine.

The other more significant negative aspect of the standard RCP testing method is the availability of the stationary phase. Because the ITLC-SG paper is being discontinued and will no longer be commercially available, it is necessary that an alternative method be developed and analyzed for the future RCP testing of ${ }^{99 \mathrm{~m}} \mathrm{Tc}$-tetrofosmin. Simply the fact that the current method is approved by the Food and Drug Administration and USP does not mean that another method is not equally good or better (9). Although the standard method is USP-approved, USP also states that an alternative method may be used as long as it is evaluated and determined to be an accurate alternative method (10).

The alternative RCP testing method is an expedited (2.3 \pm $0.1 \mathrm{~min}$ ) (4), user-friendly process that is both accurate and valid when compared with the standard ITLC method. Because the method is not as dependent on the operator's skill, obtaining accurate results is relatively simple.

Another important factor to be considered in judging both RCP testing methods is the migration sensitivity between the 2 methods. With the standard method, it is imperative that the solvent front does not exceed the premarked line on the ITLC-SG paper. Otherwise, the RCP results could be altered, producing either false-positives or false-negatives. On the other hand, the migration of the solvent front with the alternative method is not as sensitive and is not affected if the solvent front exceeds the predefined level on the strip. Therefore, the alternative method will produce accurate results more often than the current method. When considering the $86 \%$ overall specificity, one could argue that the value is too low and a cause for concern, but it is important to look at the individual specificities for each method. Although the alternative method has a specificity of $99 \%$, the standard method has a specificity of $92 \%$, indicating that the standard method has lower test-retest reliability, and therefore the overall specificity of $86 \%$ is most likely due to the imprecision of the standard method. The alternative method is a more reliable process in the fast-paced environment of nuclear medicine, which is dependent on high-quality radiopharmaceuticals and accurate RCP testing.

One concern with the alternative method involves the safety of the solvents used. There has been some concern as to whether the combination of chloroform and tetrahydrofuran might be harmful to the individual working with them (11). A possible solution to this problem could be combining the 2 ingredients in a fume hood to reduce any potential 
danger or threat that the solution could pose. Additionally, the mobile phase requires refrigeration and biweekly preparation, which can be a burdensome process.

\section{CONCLUSION}

Both the standard and alternative RCP methods have advantages and disadvantages. Yet the pros and cons of the alternative method outweigh those of the standard method, suggesting that the alternative method would be a better method for the RCP testing of ${ }^{99 \mathrm{~m}} \mathrm{Tc}$-tetrofosmin. The most important advantage of the alternative method over the standard method is the availability of the stationary phase. Also, the alternative method was more accurate in the determination of the RCP value of ${ }^{99 \mathrm{~m}} \mathrm{Tc}$-tetrofosmin and can be used to assess the RCP value of both ${ }^{99 \mathrm{~m} T c-}$ tetrofosmin and ${ }^{99 m}$ Tc-sestamibi.

\section{ACKNOWLEDGMENTS}

We thank Rose M. Busta for her assistance in the submission of this paper. We also thank Thomas J. Barna, CNMT; Robert A. Myhrvold, CNMT; and Megan Piatz, RT (R), for their assistance in our research at the Mayo Clinic Nuclear Pharmacy Laboratory. This paper was presented at the 56th Annual Meeting of the Society of Nuclear Medicine, Toronto, Canada, on June 15, 2009. Laura A. Eggert was selected by the SNM Technologist Section Awards Commit- tee to receive the student award, radiopharmacy category, for her oral presentation.

\section{REFERENCES}

1. Myoview [package insert]. Arlington Heights, IL: GE Healthcare; February 2006.

2. Technetium Tc-99m tetrofosmin injection. In: USP 32-NF27: United States Pharmacopeia and National Formulary. Rockville, MD: United States Pharmacopeial Convention; 2009:3673-3674.

3. McKay BF, Zimmer AM, Spies SM. Rapid miniaturized chromatography for technetium-99m-tetrofosmin. J Nucl Med Technol. 1997;25:52-54.

4. Hung JC, Wilson ME, Brown ML, Gibbons RJ. Rapid preparation and quality control method for technetium-99m-2-methoxy isobutyl isonitrile (technetium99m-sestamibi). J Nucl Med. 1991;32:2162-2168.

5. Pharmaceutical compounding-sterile preparations (general chapter 797). In: USP 32-NF27: United States Pharmacopeia and National Formulary. Rockville, MD: United States Pharmacopeial Convention; 2009:319-336.

6. Kowalsky RJ, Falon SW. Radiopharmaceutical chemistry. In: Radiopharmaceuticals in Nuclear Pharmacy and Nuclear Medicine, 2nd ed. Washington, DC: American Pharmacists Association; 2004:235-336.

7. Pandit-Taskar N, Grewal RK, Strauss HW. Cardiovascular system. In: Christian PE, Waterstram-Rich KM, eds. Nuclear Medicine and PET/CT Technology and Techniques. 6th ed. St. Louis, MO: Mosby; 2007:479-512.

8. Saha GB. Characteristics of specific radiopharmaceuticals. In: Fundamentals of Nuclear Pharmacy. 5th ed. New York, NY: Springer; 2004:111-150.

9. Hung JC, Ponto JA, Gadient KR, et al. Deficiencies of product labeling directions for the preparation of radiopharmaceuticals. J Am Pharm Assoc. 2004;44:30-35.

10. General notices and requirements. In: USP 32-NF27: United States Pharmacopeia and National Formulary. Rockville, MD: United States Pharmacopeial Convention; 2009:3-12.

11. Luebke AL, Wilary DM, Mahoney DW, Hung JC. Evaluation of an alternative radiochemical purity testing method for technetium-99m-sestamibi. J Nucl Med Technol. 2000;28:259-263. 